\title{
INFINITELY PERIODIC KNOTS
}

\author{
ERICA FLAPAN
}

One aspect of the study of 3-manifolds is to determine what finite group actions a given manifold has. Some important questions that one can ask about these actions on a given manifold are: What periods could they have? and, what sets of points may be fixed by the action? In the case of periodic transformations of homology spheres, Smith [18] classified the types of fixed point sets which could occur. For homology 3-spheres the fixed point set will be $\emptyset, S^{0}, S^{1}$, or $S^{2}$. Fox [4] looked at periodic transformations of the three sphere which leave a knot invariant and, using Smith's classification of fixed point sets, determined that there were eight types of transformations according to how the fixed point set met the knot. For convenience we shall say a knot is $(a, b)$-periodic if there is a periodic transformation of $S^{3}$ leaving the knot invariant with fixed point set homeomorphic to $a$ and with the fixed point set meeting the knot in a set homeomorphic to $b$. As Fox points out the possibilities for $b$ are only $\emptyset, S^{0}$, and $S^{1}$. And by the recent proof of the Smith conjecture [21] we can rule out $S^{1}$ if the knot is non-trivial.

Fox asked which knots could have infinitely many periods of each type; in other words, which knots are infinitely $(a, b)$-periodic for each possible pair $(a, b)$. Seifert [16] has shown that any knot that could be drawn on the surface of a torus is infinitely $\emptyset$-periodic (here we write $\emptyset$-periodic instead of $(\emptyset, \emptyset)$-periodic). Hartley [8] conjectured that torus knots are the only infinitely $\emptyset$-periodic knots. On the other hand, Murasugi [14] showed that any infinitely $\left(S^{1}, \emptyset\right)$-periodic knot must have trivial Alexander polynomial.

If a knot is infinitely periodic then it must either have an infinite number of orientation reversing periodic diffeomorphisms or an infinite number of orientation preserving periodic diffeomorphisms. Suppose $\left\{g_{i}\right\}$ is an infinite collection of periodic diffeomorphisms of $\left(S^{3}, K\right)$ with distinct orders $\left\{p_{i}\right\}$. Now $\left\{g_{i}^{2}\right\}$ is an infinite collection of periodic diffeomorphisms which preserve the orientation of both $S^{3}$ and $K$; and order $\left(g_{i}^{2}\right)=p_{i}$ or $p_{i} / 2$, so an infinite subcollection of $\left\{g_{i}^{2}\right\}$ have distinct orders. Now by Smith theory a periodic diffeomorphism of $S^{3}$ is orientation preserving if and only if it has a fixed point set which is empty

Received February 22, 1983, and in revised form December 20, 1983. 
or is homeomorphic to $S^{1}$. So for each orientation reversing periodic diffeomorphism $g_{i}, \operatorname{fix}\left(g_{i}\right) \neq \emptyset$. Hence fix $\left(g_{i}^{2}\right) \neq \emptyset$ and since $g_{i}^{2}$ is orientation preserving

$$
\operatorname{fix}\left(g_{i}^{2}\right) \cong S^{1} .
$$

Thus if a certain knot has infinitely many orientation reversing periodic diffeomorphisms, then it is infinitely $\left(S^{1}, \emptyset\right)$-periodic or infinitely $\left(S^{1}, S^{0}\right)$-periodic. Now suppose $g$ is a periodic diffeomorphism of $\left(S^{3}, K\right)$ with

$$
\operatorname{fix}(g) \cap K \neq \emptyset .
$$

Then $g \mid K$ is orientation reversing, and $g^{2} \mid K$ is the identity. But by the Smith conjecture [21] now $g^{2}$ itself is the identity. Now since $g_{i}^{2} \mid K$ is orientation preserving, $h_{i}{ }^{n} \mid K$ will be orientation preserving. Thus by taking $\left\{h_{i}\right\} \subseteq\left\{g_{i}^{2}\right\}$ as our infinite collection of periodic diffeomorphisms, we assure that no power of any of the $h_{i}$ fixes any point on $K$ and both $h_{i}$ and $h_{i} \mid K$ are orientation preserving.

We prove Hartley's conjecture that only torus knots are infinitely periodic, and further that no non-trivial knots are infinitely $\left(S^{1}, \emptyset\right)$-periodic. Thus no non-trivial knots have infinitely many diffeomorphisms of distinct order with non-empty fixed point set, and no non-trivial knots have infinitely many distinct order orientation reversing diffeomorphisms. Our basic strategy will be to split the knot complement along characteristic tori and to show that if a knot is infinitely periodic then all its characteristic simple and Seifert fibered components are also infinitely periodic. Then we prove that all the components are in fact Seifert fibered and we go through the remaining possibilities one at a time.

Definition 1. A knot $K$ in $S^{3}$ has a free-symmetry $h$ of order $q$, if $h$ is a fixed point free diffeomorphism of $S^{3}$ leaving $K$ invariant, and $h^{q}$ is the identity.

Definition 2. A knot $K$ in $S^{3}$ has a cyclic-symmetry $h$ of order $q$ if $h$ is a diffeomorphism of $S^{3}$ leaving $K$ invariant and

$$
\operatorname{fix}(h) \cong S^{1} \text { and } \quad \operatorname{fix}(h) \cap K=\emptyset,
$$

and $h^{q}$ is the identity.

Definition 3. $h$ is said to be a symmetry of $K$ if $h$ is a free-symmetry or a cyclic-symmetry and $h \mid K$ is orientation preserving.

We have shown in the introduction that if $K$ is infinitely periodic then $K$ has an infinite number of symmetries of distinct order.

The following three lemmas are immediate from the work of Freedman, Haas and Scott [5] and Meeks and Scott [13]. 
Lemma 1. [5]. Let $M$ be a Riemannian, Haken 3-manifold whose boundary is empty or has non-negative mean curvature. Let $\mathscr{S}$ be any collection of essential surfaces in $M$, no two of which are parallel. Then $\mathscr{S}$ is isotopic to a collection $\mathscr{T}$ of least area surfaces.

Lemma 2. [5]. Let $S_{1}$ and $S_{2}$ be least area surfaces in a Riemannian, Haken 3-manifold M. If $S_{1}$ and $S_{2}$ can be homotoped to be disjoint then $S_{1} \cap S_{2}=\emptyset$ or $S_{1}=S_{2}$.

Lemma 3. [13]. Let $M$ be a Haken 3-manifold with a $\mathbf{Z}_{p}$-action $g$. Then there exists a characteristic family of tori in $M$ invariant under $g$.

Remarks. 1) We use "characteristic" in the sense of Jaco and Shalen. Thus, if we remove the characteristic family from $M$ we are left with Seifert fibered and simple components. Also if $f: M \rightarrow M$ is a diffeomorphism then $f(T)$ is ambiant isotopic to $T$.

2) We shall want to apply Lemmas 1 and 2 to a manifold $M$ with a periodic diffeomorphism $h$ by finding a Riemannian metric for $M$ which makes $h$ an isometry and makes $\partial M$ have non-negative mean curvature. We do this as it is done in [12, p. 56].

Lemma 4. Let $k$ be a knot, and let $Q$ be its exterior in $S^{3}$. Further, let $\mathscr{T}=\left\{T_{i}\right\}$ be a characteristic family of tori in $Q$. If $k$ is infinitely periodic then $k$ has infinitely many symmetries leaving each $T_{i}$ invariant.

Proof. For each symmetry $G_{\alpha}$ of $\left(S^{3}, k\right)$ we can define $g_{\alpha}: Q \rightarrow Q$ to be a periodic diffeomorphism. Let $\mathscr{S}=\left\{S_{i}\right\}$ be the characteristic family given by Lemma 3 . Let $f_{\alpha}$ be an isotopy of $Q$ taking $\mathscr{T}$ to $\mathscr{S}$. Thus

$$
f_{\alpha}\left(T_{i}\right)=S_{i} \text { for each } i \text {. }
$$

Now

$$
f_{\alpha}^{-1} \circ g_{\alpha} \circ f_{\alpha}(\mathscr{T})=\mathscr{T}
$$

Let $h_{\alpha}=f_{\alpha}^{-1} \circ g_{\alpha} \circ f_{\alpha}$, then the order of $h_{\alpha}$ is the same as the order of $g_{\alpha}$. Let the order of $h_{\alpha}$ be $\alpha$. Since $k$ is infinitely periodic, the $\alpha$ get arbitrarily large. Let $m$ be the number of permutations there are of the elements of $\mathscr{T}$. Let $h_{\alpha}$ be a periodic diffeomorphism of $Q$ leaving $\mathscr{T}$ invariant and such that $\alpha>m$. Then for some $N_{\alpha}$ with $0<N_{\alpha} \leqq m+1, h_{\alpha}^{N_{\alpha}}$ performs the identity permutation on $\mathscr{T}$. Define

$$
H_{\alpha}=h_{\alpha}^{N_{\alpha}} \text {. }
$$

Then the order of $H_{\alpha}$ is at least $\alpha / N_{\alpha}$.

Now since the $h_{\alpha}$ have arbitrarily large orders $\alpha$, but the $N_{\alpha}$ are bounded by $m+1$, the $H_{\alpha}$ must have arbitrarily large orders. Since $h_{\alpha}$ and $h_{\alpha} \mid k$ are orientation preserving, so are $H_{\alpha}$ and $H_{\alpha} \mid k$. Hence

$$
\operatorname{fix}\left(H_{\alpha}\right) \cap k=\emptyset
$$


since by the Smith conjecture $\operatorname{fix}\left(H_{\alpha}\right) \neq k$. Thus $\left\{H_{\alpha}\right\}$ is an infinite collection of periodic diffeomorphisms of $Q$, and

$$
H_{\alpha}\left(T_{i}\right)=T_{i} \text { for each } i \text {. }
$$

Remark. Lemma 4 essentially says that if a knot is infinitely periodic then all its characteristic simple and Seifert fibered components are also infinitely periodic.

Definition 4. A 3-manifold is simple if it contains no essential torus, and a knot is simple if its exterior is simple.

Lemma 5. A simple non-torus knot is not infinitely periodic.

Proof. If $K$ is not a torus knot then $K$ is not Seifert fibered. So by [1] the center of $\pi_{1}\left(S^{3}-K\right)$ is trivial. Now by [6, Theorem] no symmetry of $\left(S^{3}, K\right)$ is pairwise isotopic to the identity. So every symmetry of $\left(S^{3}, K\right)$ induces an outer automorphism of $\pi_{1}\left(S^{3}-K\right)$ of the same order. But by Johannson's finiteness Theorem [11, page 213] Out $\left(\pi_{1}\left(S^{3}-K\right)\right)$ is finite. Hence $\left(S^{3}, K\right)$ is not infinitely periodic.

Remark. The only Seifert fibered spaces in a knot exterior are a torus knot complement, a cable space, or a composing space, as shown by Jaco and Shalen [10, Lemma VI.3.4].

Definition 5. A cable space is a manifold obtained from a solid torus $S^{1} \times D$ by removing an open regular neighborhood in $S^{1} \times \stackrel{\circ}{D}$ of a simple closed curve $C$ which lies in a torus $S^{1} \times J$ where $J$ is a simple closed curve in $D$ and where $C$ is non-contractible in $S^{1} \times D$.

Definition 6. A composing space is a 3-manifold homeomorphic to $W \times S^{1}$ where $W$ is a disk with $n$ open cells removed, for $n \geqq 2$.

Definition 7. Let $K_{2} \subseteq W \subseteq S^{3}$ be a knot which intersects every meridianal disk of a standardly embedded solid torus $W$ in the 3 -sphere. Let $K_{1}$ be another knot with a regular neighborhood $V$ in $S^{3}$. Let $h: W \rightarrow V$ be a homeomorphism preserving preferred longitude and meridian, and let $k=h\left(K_{2}\right)$. Then we say $K_{1}$ is a companion of $k$ and $K_{2}$ is a presatellite.

Definition 8. A cable knot is a knot with presatellite a torus knot.

Remark. A cable knot with companion a torus knot is one in which both $K_{1}$ and $K_{2}$ are torus knots.

LEMMA 6. If there is a non-torus knot $k$ which is infinitely periodic then either:

1. There is a cable knot with companion a torus knot which is infinitely periodic.

2. There is a composing space with incompressible boundary components $T_{0}, \ldots, T_{n}$, for $n \geqq 2$, in the complement $Q$ of a knot $k$ with $\partial Q=T_{0}$; and $k$ has infinitely many symmetries $h$ such that $h\left(T_{i}\right)=T_{i}$ for each $i$. 
Proof. Suppose $k$ has infinitely many periods. Let $Q$ be the complement of an open tubular neighborhood of $k$ in $S^{3}$. Let $\left\{T_{i}\right\}$ be a characteristic set of tori for $Q$. By Lemma $4 Q$ has infinitely many periods leaving each $T_{i}$ and hence each component $X_{l}$ of $Q-\cup T_{i}$ invariant. Since $Q$ is a knot complement every torus $T_{i}$ separates. Let $X_{1}$ be a component of $Q-\cup T_{i}$ with only one boundary component. Since $\left\{T_{i}\right\}$ is characteristic each $X_{l}$ is either simple or Seifert fibered. By [10, Lemma VI.3.4] the only Seifert fibered spaces in a knot exterior are a torus knot complement, a cable space, or a composing space. A cable space and a composing space each have more than one boundary component. So either $X_{1}$ is a torus knot complement or $X_{1}$ is simple. Now $\partial X_{1}$ is essential in $Q$ and so by [15, Proposition 3.10], $S^{3}-X_{1}=V_{1}$, a solid torus with core $K_{1}$, which is a companion of $k$. Suppose $h$ is any period of $Q$ with order greater than 2 . Then $h$ takes a meridianal disk of $V_{1}$ to a meridianal disk. So we can define a periodic diffeomorphism $\hat{h}$ of $K_{1}$ by $\hat{h}\left|X_{1}=h\right| X_{1}$ and $\hat{h}$ is defined in $V_{1}$ by extending $h$ radially. Now the order of $\hat{h}$ must be equal to the order of $h$ since $X_{1}$ cannot be the fixed point set of any periodic diffeomorphism of $S^{3}$. So the order of $\hat{h}$ is greater than 2. Thus

$$
\operatorname{fix}(\hat{h}) \cap K_{1}=\emptyset
$$

and so $\hat{h}$ is actually a symmetry of $K_{1}$.

Suppose $X_{1}$ is not a torus knot complement. Then $K_{1}$ is a simple knot other than a torus knot. But every symmetry $h$ of $k$ induces a symmetry $\hat{h}$ of $K_{1}$ of the same order. Hence we contradict Lemma 5. So $X_{1}$ is a torus knot complement. Let $\partial X_{1}=T_{1}$, and let $X_{2}$ be another component of $Q-\cup T_{i}$ with $T_{1}$ in its boundary. If no such $X_{2}$ exists then $k$ would be a torus knot. Now let the components of $Q-$ Int $X_{2}$ be a collection $\left\{R_{j}\right\}$ and $Y$, where $\partial Q \subseteq \partial Y$ and $R_{1}=X_{1}$ and $\partial R_{j} \subseteq \partial X_{2}$. Each $j$ separates $Q$ so we can let $\partial R_{j}=T_{j}$ and let $R=Q-Y$. Then $S^{3}-R=V$ a solid torus with core $K_{1}$ by [15, Proposition 3.10].

By [3, Theorem 1] there is a homeomorphism of $X_{2}$ in $S^{3}$ such that

$$
S^{3}-\operatorname{Int}\left(X_{2}\right)=\cup_{j} W_{j} \cup V^{\prime}
$$

where each $W_{j}$ and $V^{\prime}$ is a solid torus and $\partial W_{j}=T_{j}$. Let $h$ be any symmetry of $k$ leaving each $X_{1}$ and $T_{i}$ invariant. Then $h$ is orientation preserving and $h$ takes a longitude of $R_{j}$ to a longitude of $R_{j}$ for homological reasons in $R_{j}$. Also $h$ takes a meridianal disk of $S^{3}-R_{j}$ to a meridianal disk of $S^{3}-R_{j}$. Thus $h$ takes any $(p, q)$ curve on $T_{j}$ to a $\pm(p, q)$ curve on $T_{j}$. So we can define a symmetry $\hat{h}$ of $\left(S^{3}, \widetilde{K}\right)$ where $\widetilde{K}$ is the core of $V^{\prime}$. Let $\hat{h}\left|X_{2}=h\right| X_{2}$ then extend $\hat{h}$ radially to each $W_{j}$ and $V^{\prime}$. Now $\hat{h}(\widetilde{K})=\widetilde{K}$, and the order of $\hat{h}$ is equal to the order of $h$.

Now $X_{2}$ was also either Seifert fibered or simple. Suppose $X_{2}$ is not Seifert fibered. Then $X_{2}$ is simple and contains no essential annulus. So $S^{3}-$ Int $V^{\prime}$ is simple and contains no essential annulus. Thus $\widetilde{K}$ is a 
simple non-torus knot. By Lemma $5, \widetilde{K}$ has at most finitely many symmetries. But every symmetry of $k$ induces a symmetry of $\widetilde{K}$ of the same order. This contradicts $k$ having infinitely many symmetries. Hence $X_{2}$ is Seifert fibered. So $X_{2}$ is either a cable space or a composing space, since $\left|\partial X_{2}\right| \geqq 2$. If $X_{2}$ is a cable space then $R=X_{1} \cup X_{2}$, and $S^{3}-R=V$ is a neighborhood of $K$, which is a cable knot with companion a torus knot. There are infinitely many symmetries of $k$ taking a meridian of $V$ to a meridian of $V$. So for each of these symmetries $h$ we obtain a symmetry $\hat{h}$ of $K$. If $X_{2}$ is a composing space then $X_{2}$ has incompressible boundary components $T_{0}, \ldots, T_{n}$; and $T_{0}$ separates $S^{3}$ into a solid torus and the complement $Q$, of a knot $K$. As when $X_{2}$ is a cable space we can obtain infinitely many symmetries $h$ of $K$, and $h\left(T_{i}\right)=T_{i}$ for each $i$.

LEMMA 7. Let $X$ be a cable space $N_{1}$ - Int $N_{2}$ where $N_{1}$ and $N_{2}$ are solid tori in $S^{3}$, and $k$ is the core of $N_{2}$. Let $h$ be a symmetry of $\left(S^{3}, k\right)$ leaving $X$ invariant. Let $B$ be an essential annulus properly embedded in $X$ with $\partial B \subset \partial N_{2}$. Then there exists a properly embedded annulus $A$ in $X$ with $\partial A \subset \partial N_{2}$ and $h(A)=A$ and $A$ is properly isotopic to $B$ in $X$.

Remark. Recall, by our definition, if $h$ is a symmetry then $h$ and $h \mid K$ are orientation preserving.

Proof. Metrize $X$ so that $h$ is an isometry and the boundary of $X$ has non-negative mean curvature. By Lemma $1, B$ is properly isotopic to a least area essential annulus $A$. Let $l_{i}$ be a longitude and $m_{i}$ a meridian for $N_{i}$. For homological reasons in $N_{i}, h\left(m_{i}\right)$ is isotopic to $\pm m_{i}$ on $\partial N_{i}$; and for homological reasons in $S^{3}-N_{i}, h\left(l_{i}\right)$ is isotopic to $\pm l_{i}$ on $\partial N_{i}$. Now since $h$ preserves orientation on $k$ we must have $h\left(l_{2}\right)$ isotopic to $+l_{2}$. But $h$ is orientation preserving, so in fact $h\left(m_{2}\right)$ is isotopic to $+m_{2}$. Thus if $\alpha$ is any curve on $\partial N_{2}, h(\alpha)$ is isotopic to $\alpha$. In particular $h(\partial A)$ is isotopic to $\partial A$ on $\partial N_{2}$. So we could isotope $h(A)$ in $X$ to an essential annulus $E$, with $\partial E$ contained in the interior of one component $C$ of $\partial N_{2}-\partial A$. Now $E$ meets $A$ in simple closed curves in the interior of both $E$ and $A$.

Claim. We can homotop $E$ off $A$.

Proof of Claim. Isotop $E$ so that $E$ and $A$ are in general position and meet in a minimal number of components. Suppose some component $J$ of $A \cap E$ bounds a disk $D$ in $A$. Then by the incompressibility of $E, J$ also bounds a disk $D^{\prime}$ in $E$. By the irreducibility of $X, D \cup \mathrm{D}^{\prime}$ bounds a ball. Hence we could isotop $E$ to remove $J$, and thus contradict minimality. Similarly if $J$ had bounded a disk in $E$. Thus if $E \cap A \neq \emptyset$, then $E \cap C$ consists of incompressible annuli. But $C$ is homeomorphic to $T^{2} \times I$ or to a solid torus and in either case every incompressible annulus in $C$ is parallel into $\partial C$. Let $B$ be a component of $E \cap C$. Then $B$ could be pushed into $\partial C$ then further into $C-A$. Again contradicting minimality. Thus we can homotop $E$ off $A$. 
Hence we could properly homotop $h(A)$ off $A$. Now since $h$ is an isometry, $h(A)$ is also of least area. So by Lemma 2 either $h(A)=A$ or $h(A) \cap A=\emptyset$.

We show $h(A)=A$ by assuming $h(A) \cap A=\emptyset$ and deriving a contradiction. Let $W$ be a solid torus and let $Q$ be the torus knot complement

$$
Q=W \cup\left(N_{1}-\operatorname{Int} N_{2}\right),
$$

where $W$ is sewn to $\partial N$, longitude to meridian. Then by [15, Lemma 3.1] $A$ is an essential annulus properly embedded in $Q$. Let $U$ and $V$ be the closed components of $Q-A$. Then $U$ and $V$ are solid tori. Let $g: Q \rightarrow Q$ be $h \mid N_{1}$ - Int $N_{2}$ extended radially to $W$.

Case 1. Suppose there is an $i<p$ such that $g^{i}(U) \cap U \neq \emptyset$. Then either

$$
g^{i}(U) \subseteq \text { Int } U \text { for some } i,
$$

or

$$
U \subseteq \operatorname{Int} g^{i}(U) \text { for some } i .
$$

If $g^{i}(U) \subseteq$ Int $U$ then $g^{p \cdot i}(U)$. But this is a contradiction since $g^{p \cdot i}$ is the identity. Similarly, we could not have

$$
U \subseteq \operatorname{Int} g^{i}(U) .
$$

Thus Case 1 does not occur.

Case 2. For every $i<p$,

$$
g^{i}(U) \cap U=\emptyset .
$$

Let $U_{i}=g^{i}(U)$ for $i=0, \ldots, p-1$. Then the $U_{i}$ are solid tori. Let

$$
Y=V \cup_{i=1}^{p-1} U_{i}
$$

Let $B=\partial U-A$, then $B \subseteq \partial Q$. Since $g(\partial Q)=\partial Q$ we have $g(B) \subseteq \partial Q$. Now let $C=\partial V-A$. Since we are assuming that $g(A) \cap A=\emptyset$ we must have $g(B) \subseteq C$. In fact,

$$
\partial V \cap \partial U_{i}=g^{i}(B) \text { for } i=1, \ldots, p-1 .
$$

Now since $V$ is a solid torus, $Y$ is a solid torus. Now $A=Y \cap U$ is injective in $Y$ and $U$, but not surjective in $U$ since $A$ is essential in $Q$. Let $y$ generate $\pi_{1}(Y)$ and $u_{i}$ generate $\pi_{1}\left(U_{i}\right)$. Now since $g$ cyclically permutes the $U_{i}$, by the Van Kampan Theorem

$$
\pi_{1}(Q)=\left\langle y, u_{0}, \ldots, u_{p-1} \mid y^{n}=u_{0}^{m}=\ldots=u_{p-1}^{m}\right\rangle
$$


where $n \geqq 1$ and $m \geqq 2$. Thus there is an epimorphism from $\pi_{1}(Q)$ to $\oplus_{p} \mathbf{Z}_{m}$. But $S^{3} \cong W \cup N_{1}$ so

$$
Q=\operatorname{cl}\left(S^{3}-f(N)\right)
$$

where $f$ is a reembedding of $N_{2}$, and so $Q$ is a knot space. Hence

$$
H_{1}(Q) \cong \mathbf{Z} \text {. }
$$

So we have a contradiction. Thus Case 2 also does not occur.

Hence $h(A)=A$ as desired.

Remark. The proof of Case 2 is similar to that of Lemma 7.1 of [15].

THEOREM 1. Let $k$ be a cable knot with companion a torus knot K. Then $\left(S^{3}, k\right)$ is not infinitely periodic.

Proof. Let $N(k)$ be a regular neighborhood of $k$ and let

$$
Q=S^{3}-\text { Int } N(k) \text {. }
$$

Let $V$ be a regular neighborhood of $K$ containing $N(k)$, and let

$$
R=S^{3}-\text { Int } V \text {. }
$$

Since $V-$ Int $N(k)$ is a cable space it contains an essential annulus $a$ with $\partial a \subset \partial N(k)$

Now $\partial a$ separates $\partial N(k)$ into $b_{1}$ and $b_{2}$. Without loss of generality $a \cup b_{1}$ bounds a solid torus $W$ and $a \cup b_{2}$ is parallel to $\partial V$. Let $(p, q)$ be the unoriented isotopy class of $\partial a$ in $a \cup b_{2}$. Let

$$
R^{\prime}=Q-\text { Int } W .
$$

Then $R^{\prime}$ is isotopic to $R$. So $R^{\prime}$ is a torus knot complement, hence contains an essential annulus $B$ with

$$
\partial B \subset \partial R^{\prime}=a \cup b_{2} .
$$

Let $(r, s)$ be the unoriented isotopy class of $\partial B$ in $a \cup b_{2}$.

Claim. $(p, q) \neq(r, s)$.

Proof of Claim. Suppose $(p, q)=(r, s)$. Then we could properly isotope $B$ in $R^{\prime}$ so that $\partial B \cap a=\emptyset$. Since $a \subseteq \partial R^{\prime}$ in fact $B \cap a=\emptyset$; and thus $B$ is actually properly embedded in $Q$. Now $B$ and $a$ are disjoint essential annuli in $Q$. So by $[19$, Lemma 2.b] they are parallel. But $B$ was essential in $R^{\prime}$ and hence could not be parallel into $\partial R^{\prime}$. Hence $(p, q) \neq(r, s)$, as claimed.

Now suppose $\widetilde{g}$ is a symmetry of $\left(S^{3}, k\right)$. If $\left(S^{3}, k\right)$ is infinitely periodic, then without loss of generality we can assume that the order of $\widetilde{g}$ is not 2 . Now let $g=\widetilde{g}^{2}$. Then $g \mid k$ must be orientation preserving. Now $\{\partial V\}$ is a characteristic family for $Q$. So by Lemma 4 we can assume that $g(V)=V$ for an infinite class of such symmetries $g$ of $\left(S^{3}, k\right)$. Hence $g(R)=R$ and 
$g(Q)=Q$. Let $X$ be the cable space $V-$ Int $N(k)$. Then $g(X)=X$. Now by Lemma 7 there is an essential annulus $A$ properly embedded in $X$ with $\partial A \subset \partial N(k)$ and $g(A)=A$ and $A$ is properly isotopic to $a$ in $X$. Let $f_{t}$ realize this proper isotopy of $X$. We can extend $f_{t}$ to an isotopy $\hat{f}_{t}$ of $Q$. Now $\hat{f}_{1}(a)=A$. Let $C=\hat{f}_{1}(B)$, and let $R^{\prime \prime}=\hat{f}_{1}\left(R^{\prime}\right)$. Let $Q^{\prime}=Q-R^{\prime \prime}$. Then the components of $Q-A$ are the solid torus $W^{\prime}$ and the torus knot complement $R^{\prime \prime}$. Now $g(Q)=Q$ and $g(A)=A$ and $W \not R^{\prime \prime}$. Hence

$$
g\left(W^{\prime}\right)=W^{\prime} \text { and } g\left(R^{\prime \prime}\right)=R^{\prime \prime} .
$$

So $C$ is an essential annulus in the invariant torus knot complement $R^{\prime \prime}$. Now $C$ is in some cable space inside $R^{\prime \prime}$, so by Lemma 7 we can obtain an essential annulus $D$ properly embedded in $R^{\prime \prime}$ with $g(D)=D$ and $D$ is properly isotopic to $C$ in $R^{\prime \prime}$.

Observe that $(p, q)$ remains the unoriented isotopy class of $\partial A$ in $\partial R^{\prime \prime}$, and $(r, s)$ remains the unoriented isotopy class of $\partial D$ in $\partial R^{\prime \prime}$. Since $g\left(\partial R^{\prime \prime}\right)=\partial R^{\prime \prime}$, we can let $h=g \mid \partial R^{\prime \prime}$. Let $l=\operatorname{order}(g)$. By assumption we can find symmetries $g$ so that $l$ is arbitrarily large, so we can take $g^{2}$ if necessary to assure that $h$ does not switch the boundary components of $A$ or those of $D$; and

$$
h(\partial A)=+\partial A \text { and } h(\partial D)=+\partial D .
$$

Then $h$ is an order $l$ diffeomorphism of a torus $\partial R^{\prime \prime}$ which fixes setwise both a $(p, q)$ curve $\alpha \subset \partial A$ and an $(r, s)$ curve $\beta \subset \partial D$. In fact $\alpha$ and $\beta$ are simple closed curves.

Claim. There are only finitely many possible numbers $l$.

Proof of Claim. Subdivide $\beta$ into alternating $\operatorname{arcs} \gamma_{i}$ and $\delta_{i}$ such that:

1) The boundaries of $\gamma_{i}$ and $\delta_{i}$ are in $\alpha \cap \beta$.

2) Each $\gamma_{i}$ together with an arc of $\alpha$ is null homotopic in $\partial R^{\prime \prime}$.

3) Each $\gamma_{i}$ is maximal with respect to the above properties.

Let $N$ be the minimal number of points of intersection of a $(p, q)$ curve and an $(r, s)$ curve. Then

$$
N=\sum_{i}\left|\operatorname{Int} \delta_{i} \cap \alpha\right|
$$

Observe that by property 2) above, $h\left(\left\{\gamma_{i}\right\}\right)=\left\{\gamma_{i}\right\}$ and hence

$$
h\left(\left\{\delta_{i}\right\}\right)=\left\{\delta_{i}\right\} .
$$

So $h$ permutes the set $\cup_{i}$ Int $\delta_{i} \cap \alpha$. Note that since $(p, q) \neq(r, s), N>0$. Thus either $l$ divides $N$ or there is an $i<l$ such that

$$
h^{i}(x)=x \text { for some } x \in \cup \text { Int } \delta_{i} \cap \alpha .
$$

Recall that $h \mid \alpha$ and $h \mid \beta$ preserve orientation. So, in fact, $h^{i} \mid(\alpha \cup \beta)$ is the identity. But now $g^{i}$ is an orientation preserving periodic diffeomorphism 
of $S^{3}$ yet

$$
\alpha \cup \beta \subseteq \operatorname{fix}\left(g^{i}\right) \text {. }
$$

This contradicts Smith Theory. Thus $l$ divides $N$ and so there can be only finitely many $l$ 's.

Thus $\left(S^{3}, k\right)$ has only a finite number of symmetries.

LEMMA 8. Let $X_{2}$ be a composing space with incompressible boundary components $T_{0}, T_{1}, \ldots, T_{n}$ in $Q$, the complement of a knot $k$, with $\partial Q=T_{0}$ and $n \geqq 2$. Then $K$ has no symmetry $h$ of order greater than 2 such that $h\left(T_{i}\right)=T_{i}$ for each $i$.

Remark. The reader should recall that by our definition if $h$ is a symmetry, then $h$ and $h \mid K$ are orientation preserving.

Proof of Lemma 8. Assume there is such a symmetry $h$. Let $g=h^{2}$, and let $l_{i}$ and $m_{i}$ be a longitude and meridian of $T_{i}$, respectively. Further, let $T_{i}$ separate $S^{3}$ into a solid torus $W_{i}$ and a knot complement $R_{i}$. Then $g$ takes a surface in $R_{i}$ bounded by $l_{i}$ to a surface in $R_{i}$ bounded by $g\left(l_{i}\right)$. Since $g=h^{2}$ we can in fact assure that $g\left(l_{i}\right)$ is isotopic to $+l_{i}$ on $T_{i}$. Now if $n \geqq 3$ extend $g$ radially within a solid torus $U_{i}$ replacing $R_{i}$ for $i=3, \ldots, n$. The original knot $K$ was a composite knot with $n$ components. By replacing these $R_{3}, \ldots, R_{n}$ by $U_{3}, \ldots, U_{n}$ we have created a new manifold, $X$, which is the complement of a composite knot $k$ with two components. Now $g$ is a symmetry of $W \times S^{1}$ where $W$ is a disk with two holes. Metrize $X=W \times S^{1}$ so that $h$ is an isometry and $\partial X$ has non-negative mean curvature. Let $A$ be an essential annulus properly embedded in $X$ with both boundary components in $T_{1}$. By Lemma 1 find a least area surface $B$ isotopic to $A$ in $X$. Now $B$ separates $X$ into components $Y_{1}$ and $Y_{2}$ which are each homeomorphic to $S^{1} \times S^{1} \times I$, and $T_{i} \subseteq Y_{i}$. Now $Y_{i}$ contains no essential annulus with both boundaries in $T_{0} \cup B$, so we can homotop $g(B)$ disjoint from $B$. So by Lemma 2,

$$
g(B)=B \text { or } g(B) \cap B=\emptyset .
$$

We show $g(B)=B$. Suppose $g(B) \cap B=\emptyset$. Then

$$
g\left(Y_{1}\right) \subseteq \text { Int } Y_{1} \quad \text { or } \quad Y_{1} \subseteq \operatorname{Int} g\left(Y_{1}\right)
$$

since $T_{2}=g\left(T_{2}\right) \subseteq g\left(Y_{1}\right)$. But if $g\left(Y_{1}\right) \subseteq$ Int $Y_{1}$ and let $\rho=\operatorname{order}(g)$ then

$$
g^{\rho}\left(Y_{1}\right) \subseteq \text { Int } Y_{1}
$$

and this implies that $Y_{1} \subseteq$ Int $Y_{1}$. By this contradiction we conclude that $g(B)=B$ as desired.

Now by [9, Theorem VI.34] $B$ is saturated in some Seifert fibration of $X$. So by [15, Lemma 6.4] each component $b_{i}$ of the boundary of $B$ is a meridian of the solid torus $V=S^{3}-Q$ with core $K$. Now since $p>2, g$ is 
not the identity, and since $g-h^{2}$, then $g\left(b_{i}\right)=b_{i}$ for each component $b_{i}$ of $\partial B$. But $b_{i}$ is a meridian of $V$, so $g\left(b_{i}\right)=b_{i}$ implies that $g$ fixes a point on $k$. But $g \mid k=h^{2}=k$ is orientation preserving. Thus $g$ can only fix a point of $k$ if $g \mid k$ is the identity. But this contradicts the Smith Conjecture [21]. So we could not have had such a symmetry $h$ to begin with.

THEOREM 2. No knots other than torus knots could have infinitely many symmetries with distinct orders.

Proof. By Lemma 6 if there were an infinitely periodic knot other than a torus knot then either

1) There is a cable knot with companion a torus knot which is infinitely periodic, or

2) There is a composing space $X_{2}$ with incompressible boundary components $T_{0}, \ldots, T_{n}, n \geqq 2$; and $X$ is in the complement $Q$ of a knot $k$ with $\partial Q=T_{0}$. Further $K$ has a symmetry $h$ of order at least 3 and $h\left(T_{i}\right)=T_{i}$ for each $i$.

However, case 1) is ruled out by Theorem 1 and Case 2 is ruled out by Lemma 8. Thus neither case can occur.

THEOREM 3. The only knots with infinitely many distinct order free-symmetries are torus knots. No non-trivial knots have infinitely many distinct order cyclic-symmetries.

Proof. Conner [2, Theorem 4.3] showed that torus knots have only finitely many cyclic-symmetries. Whereas, Seifert [16] proved that torus knots have infinitely many free-symmetries.

Hence, as pointed out in the introduction, we have also shown that no non-trivial knots have infinitely many orientation reversing periodic diffeomorphisms, and further no non-trivial knots have infinitely many periodic diffeomorphisms with non-empty fixed point set.

\section{REFERENCES}

1. G. Burde and H. Zieschang, Eine Kennzeichnung der Torus knoten, Math. Ann. 167 (1966), 169-176.

2. P. E. Conner, Transformation groups on a $K(\pi, 1)$, II, Michigan Math. J. 6 (1959), 413-417.

3. R. H. Fox, On the imbedding of polyhedra in 3-space, Ann. of Math. 49 (1948), $462-470$.

4. Knots and periodic transformations, Proc. The Univ. of Georgia Inst. (PrenticeHall, Englewood Cliffs, N.J., 1961), 120-167.

5. M. Freedman, J. Haas and P. Scott, Lease area incompressible surfaces in 3-manifolds, to appear in Inventiones Mathematicae.

6. C. H. Giffen, On transformations of the 2-sphere fixing in a knot, Bull. Amer. Math. Soc. 73 (1967), 913-914.

7. R. I. Hartley, Knots and involutions, Math. Z. 171, (1980), 175-185.

8. Knots with free period, Can. J. Math. 33 (1981), 91-102. 
9. W. Jaco, Lectures on three-manifold topology, Memoirs AMS 43 (1980).

10. W. Jaco and P. Shalen, Seifert fibered spaces in 3-manifolds, Memoirs AMS (1979).

11. K. Johannson, Homotopy equivalences of 3-manifolds with boundaries, Lecture Notes in Mathematics 761 (1979).

12. W. Meeks, A survey of the geometric results in the classical theory of minimal surfaces, Bol. Soc. Bras. Mat. 12 (1981), 29-86.

13. W. Meeks and P. Scott, Finite group actions on 3-manifolds, preprint.

14. K. Murasugi, On periodic knots, Comment. Math. Helv. 46 (1971), 162-174.

15. R. Myers, Companionship of knots and the Smith conjecture, Trans. Amer. Math. Soc. 259 (1980), 1-32.

16. H. Seifert, Topologie dreidimensionalen gefaserter Räume, Acta Math. 60 (1933), 147-238.

17. J. Simon, An algebraic classification of knots in $S^{3}$, Ann. of Math. 97 (1973), 1-13.

18. P. A. Smith, Transformations of finite period II, Ann. of Math. 40 (1939), 690-711.

19. G. A. Swarup, P. A. Smith conjecture for cable knots, Quart. J. Math. Oxford 31 (1980), 105- 108

20. F. Waldhausen, Gruppen mit Zentrum und dreidimensionale Mannigfaltigkeiten, Topology 6 (1967), 505-517.

21. Proceedings of the 1979 Conference on the Smith Conjecture at Columbia University, to appear.

Rice University,

Houston, Texas 